\title{
Augmented In-Band Telemetry to the User Equipment for Beyond 5G Converged Packet-Optical Networks
}

\author{
D. Scano(1), F. Paolucci(1,2), K. Kondepu(1), \\ A. Sgambelluri(1), L. Valcarenghi(1), P. Castoldi(1), F. Cugini(2)
}

(1) Scuola Superiore Sant'Anna, Pisa, Italy, davide.scano@santannapisa.it

(2) CNIT, Pisa, Italy

\begin{abstract}
Traffic monitoring through in-band telemetry is extended up to the User Equipment (UE), providing accurate e2e latency measurement. The UE becomes aware of its experienced service performance, enabling autonomous operations for faster automatic source-based Edge-Cloud steering.
\end{abstract}

\section{Introduction}

In the 5G architecture, latency critical applications are supported through a converged packet optical infrastructure that ranges from the cloud to the edge computing resources. In this context, P4-based In-band Network Telemetry (INT) has emerged as a candidate technology enabling the monitoring of the actual edge-cloud experienced latency ${ }^{[1-3]}$. In case of critical service conditions, the SDN Controller (/Orchestrator) is typically involved to recover adequate service conditions.

Beyond $5 G$ (B5G) is now calling for a fully endto-end (e2e) architecture, where the infrastructure is extended up to the B5G user equipment (UE) ${ }^{[4]}$. In addition, B5G aims at providing self-management of (pre-planned) services, thus avoiding the SDN controller intervention during critical events while minimizing control overhead.

In this work we propose to extend P4 INT up to the 5G user equipment and perform INTtriggered source-based steering to the most suitable cloud-edge node satisfying the required service latency. The solution resorts to extended P4-based INT programmability and is implemented and evaluated in a hybrid multisegment testbed including a real wireless domain, a packet-switched backhaul and an SDN disaggregated metro-core optical domain running optical telemetry against soft failure events.

\section{INT in 5G converged packet-optical network}

Fig. 1 shows the network scenario motivating the proposed augmented INT system. A 5G network is connected to a cloud data center by means of a backhaul and metro-core optical network with P4 programmable bare metal switches (i.e., N1N6 in Fig. 1) running in-band telemetry (INT). Services offered to $5 \mathrm{G}$ users are deployed on cloud resources and closer edge node resources $E 1$ and $E 2$, depending on service requirements in terms of bounded latency.

Typically, INT is programmed at each switch to

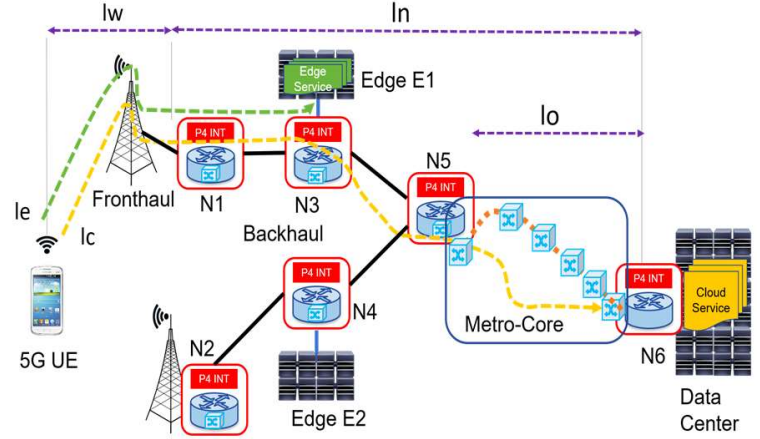

Fig. 1: $5 \mathrm{G}$ Edge Cloud traffic in INT-enabled SDN wired network: monitored and unmonitored latencies.

push custom headers to selected traffic flows, for example conveying information on the actual latency spent by packets within each node (i.e., hop latency). Thus, INT is capable of monitoring intra-switch latencies. Instead, link latencies may not be retrieved since they would require synchronization among all nodes, typically not available in an e2e scenario with multiple domains. In a fully packet-switched wired network such approach is appropriate to detect latency variations, typically occurring inside the switch due to congestion, queueing and buffering. However, in a wired-wireless e2e path including multi-layer optical networks, significant link latency variations may occur, not monitored by INT. First, in a multi-layer network, a working lightpath in the optical layer may be re-routed along a longer distance path (e.g., upon recovery or optimization), thus increasing link latency without affecting the IP layer end points. For example, soft failures detected by optical telemetry may trigger the optical SDN controller to exclude a compromised optical link and reconfigure the lightpath to a longer backup path [5]. Second, the Radio Access Network (RAN) system (i.e., wireless links and different functional split among Distributed -DU and Centralized Unit $-\mathrm{CU}$ ), is subject to latency variations due to mobility, users subscription, queueing delay, frame alignment and transmission processing ${ }^{[6]}$. This means that, in the case of wired-wireless 


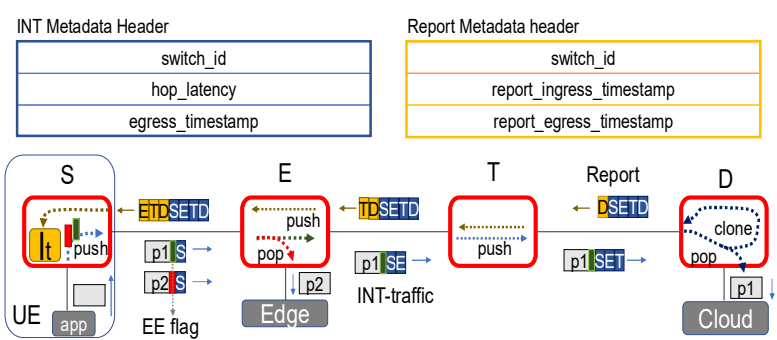

Fig. 2: INT/Report Headers pushed at each node,

Augmented INT and source-based cloud-edge steering.

connectivity including optical domains, standard INT is unaware of the e2e latency and is not able to guarantee latency-bounded service monitoring.

Following Fig. 1, UE service towards the cloud experiences excessive latency $\mathrm{I}_{\mathrm{c}}$, however the INT system is able to monitor only the intra-switch latencies of the N1-N6 segment, without detecting any significant issue. However, lo latency in the metro-core network may change due, e.g., to an optical lightpath recovery in the N5-N6 link, increasing the overall wired network segment latency $\ln$. In addition, the RAN wireless and fronthaul link is introducing additional unmonitored Iw latency. The service might be steered to path N2-N6, thus requiring handover, however In would remain high. Alternatively, the same traffic might be consumed by the local Edge node E1, experiencing a reduced and allowed latency $\mathrm{l}_{\mathrm{e}}<\mathrm{l}_{\mathrm{c}}$. Unfortunately, the INT domain is unaware of both $l_{0}$ and $l_{w}$ thus leaving the UE with an e2e unmonitored service delay.

\section{Proposed augmented INT and source-based cloud-edge steering}

To enable B5G e2e monitoring and dynamic cloud-edge steering without involving the controller, we propose to augment the P4-based INT system including the mobile user equipment (UE) in the INT domain. The solution includes three main novelties: a) UE inclusion in the INT domain, b) extended handling of the INT Report packet to compute link latencies and c) automatic source-based Edge-Cloud steering.

First, a programmable switch is implemented into the UE as a service app (e.g., under the form of a lightweight virtual container) and programmed by the Orchestrator to act as INT source node. Referring to upload service traffic, as shown in Fig. 2, INT is programmed between source (S), transit (T) (including the Edge switch $E$ ) and destination (D) nodes. Node $S$ is co-located with the UE, adding its own INT headers. Node $D$ is co-located with the data center and removes all INT headers providing traffic transparently to the server. Similarly, E nodes are programmed to pop INT headers only to traffic steered towards the Edge.

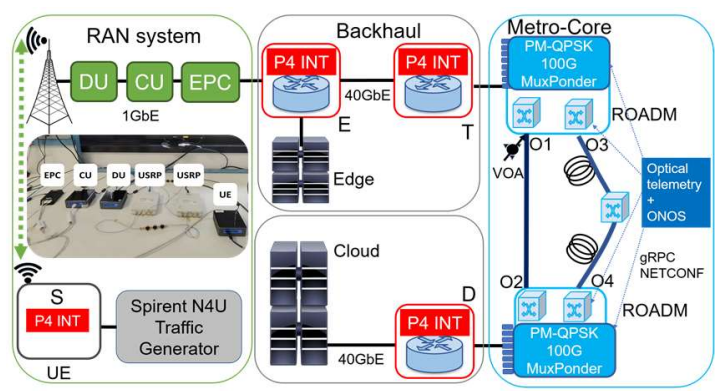

Fig. 3: Experimental network testbed.

Second, differently with respect to standard INT, node $\mathrm{D}$ is programmed to recirculate a Report traffic packet in the backward direction up to $S$. The Report packet, traditionally destined to the SDN controller via a control plane port, besides INT information collected in the forward direction (e.g., hop latencies), is extended to be pushed with the ingress and egress timestamps measured at each node in the backward direction resorting to extended INT P4 code. This allows to compute link latencies. Since P4 switches timestamps are referred to the local P4 switch bootstrap time, latencies need to be computed referring to differential times related to the same switch. When the Report reaches $S$, the switch is able to compute also the wireless link latency and stores the total e2e latency $\mathrm{I}_{\mathrm{t}}$ in a $\mathrm{P} 4$ register.

Third, service traffic generated by the UE is INTtagged by $S$ with a novel INT shim header flag called EnableEdge (EE). The flag indicates whether $\mathrm{I}_{\mathrm{t}}$ stored in $\mathrm{S}$ exceeds a pre-determined bound latency threshold (Th) imposed by the service. The flag is inspected only by $E$ node. In the case the flag is active, E automatically pops INT headers and steers traffic to the edge. This way, the UE performs source-based steering imposing traffic to reach the closer edge when $I_{t}>$ Th, without involving controllers. To avoid instabilities, EE is activated when a predetermined number of packets experience $\mathrm{l}_{\mathrm{t}}>\mathrm{Th}$, utilizing a further occurrence P4 counter.

\section{Experimental Demonstration}

The proposed augmented P4 INT has been implemented and evaluated in a hybrid wiredwireless network testbed, shown in Fig. 3. The wired backhaul network segment is composed by 40 Gigabit Ethernet interfaces and a stack of Linux box P4 switches running Behavioral Mode version 2 (BMv2) and implementing augmented INT P4 code over INT code version 2. $1^{[7]}$. The metro-core segment includes a pair of disaggregated ROADMs equipped with Ericsson PM-QPSK 100G muxponders and two Finisar WSS each, acting as configurable degrees. Two bidirectional optical paths $\mathrm{O} 1-\mathrm{O} 2$ and $\mathrm{O} 3-\mathrm{O} 4$ may 


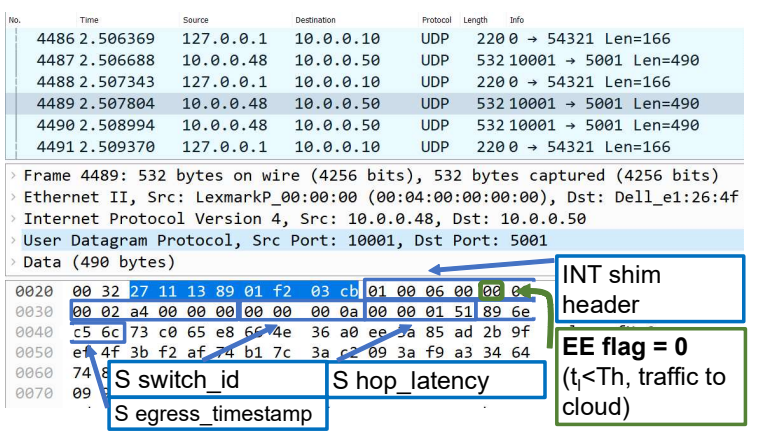

Fig. 3: Wireshark capture at the UE (switch S): INT headers

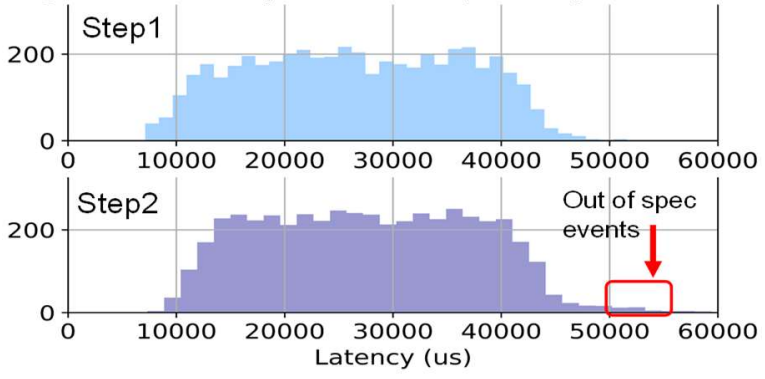

Fig. 5: RAN latency distribution (link S-E)

be activated. Primary path $01-02$ is $1 \mathrm{~km}$ long, backup path $\mathrm{O} 3-\mathrm{O} 4$ is a geographical multi-span link. The ROADMs are handled by a ONOSbased SDN controller running NETCONF/YANG API and gRPC optical telemetry services monitoring the BER and the OSNR at the muxponder cards ${ }^{[8]}$. The RAN components - UE, $\mathrm{DU}, \mathrm{CU}$ are deployed in NUC mini-PC and the Evolved Packet Core (EPC) is deployed in UPBoard mini-PC (see Fig. 3). The OpenAirlnterface $(\mathrm{OAI})^{[0]}$ open-source software is considered for the RAN deployment, and the radio hardware units are prototyped using Ettus B210. The Option 2 functional split with 25 resource blocks and single-input-single-output is considered for the DU-CU interface. The UE runs BMv2 as a container to act as source INT node S. Upload service traffic, provided by the Spirent N4U traffic generator co-located at the UE, is a constant bit rate UDP flow towards the cloud server. INT is initially activated between $S$ and $D$ through the SE-T-D path to steer traffic to the cloud server. E2e service latency threshold Th is set to $50 \mathrm{~ms}^{[10]}$.

The evaluation considers three link latency variation steps. In step 1 the service is connected to the cloud using 01-O2 optical path (telemetrymonitored BER $1.9 \times 10^{-8}$, OSNR $38.5 \mathrm{~dB}$ ) and the RAN is considered without secondary services. In step 2, fronthaul traffic coexistence with additional secondary services ${ }^{[11]}$ is considered, which further introduces an additional latency in the e2e link. Secondary services are emulated by introducing iperf traffic (i.e., $600 \mathrm{Mb} / \mathrm{s}$ ) in the DU$\mathrm{CU}$ interface. In step 3 , including the RAN latency of step 2, a variation of the VOA at $\mathrm{O} 1$ induces a soft failure in $01-\mathrm{O} 2$ causing a degraded $10^{-2}$

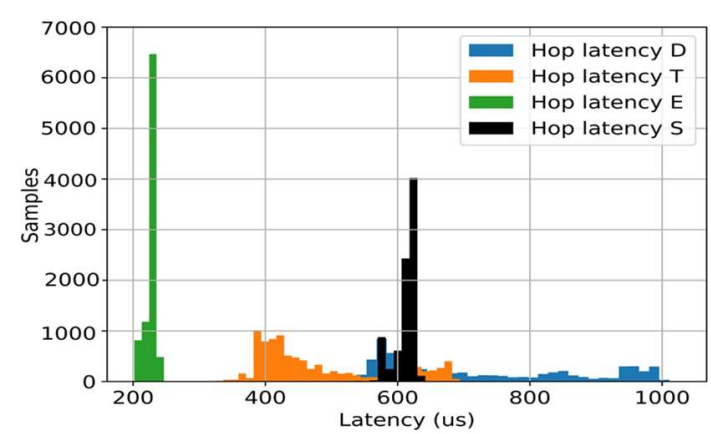

Fig. 4: BMv2 hop latencies distribution

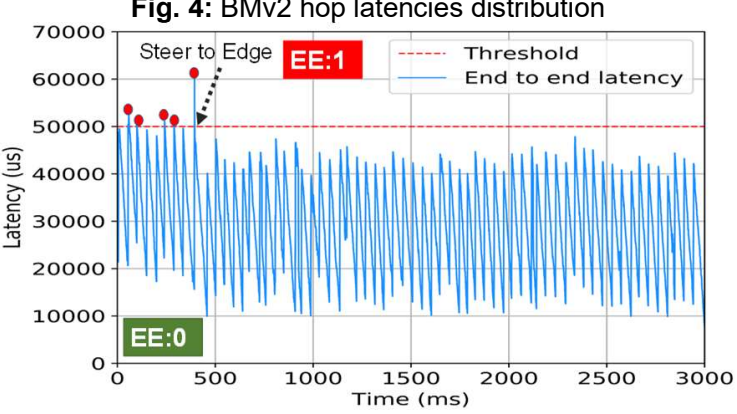

Fig. 6: E2E latency in step 3: cloud-edge steering event.

BER. The optical telemetry notifies ONOS triggering make- before-break restoration to O3$\mathrm{O} 4$ avoiding hard failures, however originating a further latency increase in the optical domain. Fig. 3 shows a Wireshark capture of INT-tagged traffic packets at the UE (source IP 10.0.0.48), showing the shim header with the novel EE flag and the metadata header pushed by S. Report packet are also received by $S$ (frames recirculated by $D$, source IP 127.0.0.1). Fig. 4 reports the monitored BMv2 hop latencies, all within $1 \mathrm{~ms}$, where $S$ and $D$ experience improved INT processing. Fig. 5 shows the RAN latency distribution in step 1 (always below 50ms) and step 2 (sporadic out-of-spec events exceeding $50 \mathrm{~ms}$ ). Such critical latencies are monitored directly by the UE traffic itself. Fig. 6 depicts the monitored e2e latency for a flow sequence (1 packet $/ \mathrm{ms}$ ) in step 3 , after optical restoration. The figure shows a high number of above Threshold events that trigger, at around time $t=500 \mathrm{~ms}$, the EE flag activation at $S$. Then, traffic is steered to the edge experiencing a reduction of the average latency of around $6 \mathrm{~ms}$, due to the exclusion of the edge-cloud segments, successfully guaranteeing in-spec e2e latency for all the flow packets.

\section{Conclusions}

A novel P4 augmented INT solution providing detailed e2e latency to the UE is proposed and evaluated in a converged packet-optical network including RAN. Results showed the effectiveness of the system to steer cloud traffic to the edge upon out-of-spec e2e latency without any controller intervention. Results also highlight the novel key feature of monitoring RAN latencies up to the UE for future B5G deployments. 
Acknowledgements: This work has received funding from the ECSEL Joint Undertaking (JU) BRAINE Project, under grant agreement No 876967. The JU receives support from the European Union's H2020 research and innovation programme and from Italy Ministry of Education, University and Research (MIUR).

\section{References}

[1] S. Tang, J. Kong, B. Niu and Z. Zhu, "Programmable Multilayer INT: An Enabler for Al-Assisted Network Automation," in IEEE Communications Magazine, vol. 58, no. 1, pp. 26-32, January 2020.

[2] I. Pelle, F. Paolucci, B. Sonkoly, F. Cugini, "Telemetrydriven Optical 5G Serverless Architecture for LatencySensitive Edge Computing", OFC 2020, paper M1A.1.

[3] F. Cugini, P. Gunning, F. Paolucci, P. Castoldi, A. Lord, "P4 In-Band Telemetry (INT) for Latency-Aware VNF in Metro Networks", OFC 2019, paper M3Z.6.

[4] K. Samdanis, T. Taleb, "The Road beyond 5G: A Vision and Insight of the Key Technologies," in IEEE Network, vol. 34, no. 2, pp. 135-141, March/April 2020, doi: 10.1109/MNET.001.1900228.

[5] F. Paolucci, A. Sgambelluri, F. Cugini and P. Castoldi, "Network Telemetry Streaming Services in SDNBased Disaggregated Optical Networks," in Journal of Lightwave Technology, vol. 36, no. 15, pp. 3142-3149, 1 Aug.1, 2018.

[6] I. Parvez, A. Rahmati, I. Guvenc, A. I. Sarwat and H. Dai, "A Survey on Low Latency Towards 5G: RAN, Core Network and Caching Solutions," in IEEE Communications Surveys \& Tutorials, vol. 20, no. 4, pp. 3098-3130, Fourthquarter 2018.

[7] "In-band Network Telemetry (INT) Dataplane Specification", version 2.1, P4.org Applications Working Group, 2020, https://github.com/p4lang/p4applications/blob/master/docs/INT_v2_1.pdf.

[8] A. Sgambelluri, A. Giorgetti, F. Paolucci, P. Castoldi, $F$. Cugini, "Open Source Implementation of OpenConfig Telemetry-Enabled NETCONF Agent", 2019 21st International Conference on Transparent Optical Networks (ICTON), 2019.

[9] N. Nikaein, M. K. Marina, S. Manickam, A. Dawson, R. Knopp, and C. Bonnet, "OpenAirlnterface: A Flexible Platform for 5G Research", SIGCOMM Comput. Commun. Rev. 44, 5 (October 2014), 33-38.

[10] 3GPP TR 25.913, "Requirements for further advancements for Evolved Universal Terrestrial Radio Access (E-UTRA) (LTE-Advanced)", v16.0.0, July, 2020.

[11] A. O. Mufutau, F. P. Guiomar, M. A. Fernandes, A. Lorences-Riesgo, A. Oliveira and P. P. Monteiro, "Demonstration of a hybrid optical fiber-wireless 5G fronthaul coexisting with end-to-end $4 \mathrm{G}$ networks," in IEEE/OSA Journal of Optical Communications and Networking, vol. 12, no. 3, pp. 72-78, March 2020. 